\title{
Development of a Chemical Health Risk Assessment Tool for Health Risk Assessment from Exposure to Hazardous Chemicals
}

\author{
Mohanad El-Harbawi ${ }^{1 *}$, Aira Amira Abd Raman ${ }^{2,3}$ and Fahad Al-Mubaddel ${ }^{1,4}$ \\ ${ }^{1}$ Department of Chemical Engineering, King Saud University, Saudi Arabia \\ ${ }^{2}$ Chemical Engineering Department, Universiti Teknologi PETRONAS, Malaysia
}

${ }^{3}$ PETRONAS Carigali Sdn. Bhd., Sarawak Operations, Sarawak

${ }^{4}$ Fellow, King Abdullah City for Renewable and Atomic Energy: Energy Research and Innovation Center, Saudi Arabia

*Corresponding author: Mohanad El-Harbawi, Department of Chemical Engineering, King Saud University, Riyadh, 11421, Saudi Arabia

\section{ARTICLE INFO}

Received: 慧 June 16, 2020

Published: 慧 June 29, 2020

Citation: Mohanad El-Harbawi, Aira Amira Abd R, Fahad Al-Mubaddel. Development of a Chemical Health Risk Assessment Tool for Health Risk Assessment from Exposure to Hazardous Chemicals. Biomed J Sci \& Tech Res 28(3)-2020. BJSTR. MS.ID.004669.

Keywords: Hazardous Chemicals; Human Health; Risk Assessment; Computer Tool; Visual Basic

\section{ABSTRACT}

People are exposed to chemicals on a daily basis, which can cause health disorders or even fatalities depending on their dosages. This study was conducted to develop a chemical health risk assessment (CHRA) tool to assess the risk to human health from exposure to hazardous chemicals. For developing this tool, the United States' National Academy of Sciences (US-NAS) risk assessment paradigm and the Malaysian standards and regulations (guidelines for the assessment of the health risks arising from the use of hazardous chemicals in the workplace) were used as a framework for estimating the risk from exposure to hazardous chemicals. The computer program is written and designed within an object-oriented framework using Visual Basic (VB) programming language. The tool is designed to work as a stand-alone user-friendly software. Moreover, this tool is supported with some useful options such as data for permissible exposure limit (PEL) for common chemicals, odor level thresholds, and solvent drying time to provide users with some knowledge about the materials. Furthermore, it has an internal help function to assist users on how to use the tool. The software is also designed in such a manner that whenever the user makes a mistake while using the application, an error message will be prompted on the fault. This makes the CHRA tool easy for users to identify the risk associated due to exposure to hazardous chemicals and assess the adequacy of the control measures. The CHRA software is designed to be compatible with all Windows operating systems.

\section{Introduction}

Since the past several decades, chemicals have undoubtedly played an essential role in human activities. In addition, large quantities of chemicals have been manufactured, and currently new chemicals are still being introduced every day [1]. People are exposed daily to a variety of substances that are not hazardous under usual circumstances. However, when any substance that comes into contact with or enters the body, it becomes harmful at a certain level of exposure and can be tolerated by the human body for certain limits of exposure without causing any health effect $[2,3]$.
Exposure to chemical substances can occur via ingestion, injection, and skin absorption. However, the most common route of entry in occupational and nonoccupational environments is via inhalation $[2,4,5]$. Although an appropriate usage of chemical substances has significantly contributed toward improving our quality of life, their misuse may cause serious health problems and even death. During the past several decades, there have been a series of major accidents worldwide, resulting in numerous deaths, economic losses, and environmental damage. Human exposure to a variety of 
chemical substances and the subsequent poisonings and fatalities have become a major global concern and is currently receiving significant international attention [6]. The World Health Organization (WHO) has estimated that 193,460 people have died worldwide due to unintended poisoning [7].

Several approaches have been endeavored to make our outdoor environment cleaner and safer. The WHO member states in the European region at the ministerial conference on environment and health, which was held in Budapest (2004), agreed that action should be taken without delay to reduce the possible effects of chemicals on human health. Appropriate management of chemicals can minimize the hazards of these chemicals [8]. Health risk assessment provides a systematic assessment of factors that may cause harmful impacts on human health by identifying, quantifying, and communicating the risk and the potential hazards or harm [9-11]. During the 1970s, risk assessment procedures for all chemicals were evaluated and modified, and most importantly, formalized [12]. A part of this development was the initiation of the WHO Environmental Health Criteria Programme in 1973. The first environmental health standards study on mercury was published in 1976, and since then, an increasing number of assessments of chemicals and their physical effects have been produced [13]. Risk assessment implies a careful examination and a systematic method of organizing anything in the workplace that can harm people such as known and unknown information about toxicity of chemicals and interpretation of data as a basis for risk-regulatory decisions $[9,14]$. In conducting risk assessments, the United States' National Academy of Sciences (US-NAS) risk assessment paradigm has been widely used as a framework for predicting the risk from exposure to environmental chemicals [15]. This paradigm divides the risk assessment process into four steps as follows:

1) Hazard identification,

2) Hazard characterization,

3) Exposure assessment, and

4) Risk characterization. Risk assessment, which consists of these elements, is currently recognized as an essential tool by several well-known international bodies, and it is also recognized as a process that is constantly evolving and that has changed significantly in the past few decades [15-21].

Risk assessment tools and methodologies help organizations in assessing their risks. Familiar health risk assessment tools include checklists, a useful tool in identifying hazards, guidance documents, handbooks, brochures, questionnaires, and computerized tools [22]. Using computerized tools implies using a software application for conducting risk assessment. This method uses the qualitative or quantitative approach or a combination of both with the help of analytical tools to analyze and estimate the risks. To assess the risk of exposure to hazardous chemicals, the first step required is to obtain the hazard information of the chemicals. Chemical safety data sheet (CSDS) provides useful information such as the hazard description, potential hazards of the material, toxicological information, and the potential environmental effects. Mathematical equations can be used to estimate the risk to health from exposure to hazardous chemicals. They consist of sets of equations and assumed data on the chemical source. In this paper, we present the development methodology of a chemical health risk assessment (CHRA) tool that can be used to assess the risk to health from exposure to hazardous chemicals within the workplace. This tool was developed based on the Malaysian standards and regulations (guidelines for the assessment of the health risks arising from the use of hazardous chemicals in the workplace) [23] and the US-NAS risk assessment paradigm [15]. The mathematical equations and necessary data were integrated into the developed tool using the VB programming language.

\section{Materials and Methods}

The methodology of this work is divided into two stages; the first stage involves collection of important data, including hazard information of the chemicals and the necessary calculations required for determining the exposure limit. These data were measured using the current Malaysian standards and regulations and incorporated into the developing tool. The second stage involves the development of the software. A summary of the necessary information and its sources used in this work were obtained from the Malaysian standards and regulations (Guidelines for the Assessment of the Health Risks arising from the use of Hazardous Chemicals in the Workplace, second edition:

http://www.dosh.gov.my/images/dmdocuments/glx/garispanduan27.pdf) [23].

\section{Risk Assessment Steps}

As mentioned in the introduction section, the risk assessment process can be divided into four distinct steps [15] as follows:

Hazard Identification: Hazard identification is the first stage in risk assessment and involves the collection and evaluation of data on the types of health diseases and the effects resulting from the impact of chemicals [24]. The hazards associated with any chemical can be determined by simply knowing the concentration and the class of the chemical, and then the risk associated with this chemical can be determined by referring to standard reference sources for data and guidelines regarding toxicity, flammability, and other hazards $[25,26]$. Hazard information can be found in several different sources such as the material safety data sheets (MSDS). Based on the information provided in the MSDS, the hazard of any material can be assessed and the hazard rating can be designated.

Exposure Assessment: Exposure assessment is the primary step in the risk assessment process because, without exposure, even the most toxic chemicals cannot pose a threat [26]. Exposures 
are evaluated by assessing the potential for contact with the hazardous chemical, the route of exposure, the frequency and duration of exposure, and the intensity of each exposure. The longer the use of the hazardous chemical, the greater is the exposure [23]. To assess the degree of exposure appropriately, it is necessary to make a distinction between chronic and acute exposure and toxicity. Acute toxicity describes the adverse effects caused from a single and short exposure. These effects generally occur quickly and are often reversible. To assess the likelihood of the occurrence of acute effects, it is necessary to determine the frequency of exposure. Chronic toxicity describes the adverse effects caused from repeated exposures over a prolonged period of time. The effects are generally delayed and are often irreversible.

Risk Characterization: Risk is a function of both hazard and exposure. The risk characterization phase predicts the harmful effects on human health caused by a toxic substance and outlines the permissible exposure levels from which exposure standards are set. It includes collecting the information obtained in previous steps for decision-making.

Risk Management: After the completion of the risk assessment of the chemical, the risk management process follows that includes assessment of the adequacy of the control measures. Risk management decisions should take into account the identification, quantification, and communication of risk, which are determined by risk assessments [27]. The risk management process consists of two factors, the hazard posed by the activity and the probability of the occurrence of the hazard. When both the potential hazard and the probability are low, the decision will be "very low risk." However, when both the probability and the hazard are high, the decision will be "do not take the risk of the proposed action". Difficulty arises when one factor is high and the other is somewhat low [28]. Control measures imply the actions that should be taken to prevent or reduce the occurrence of risks. These control procedures may include the following steps:

(i) The substitution of hazardous chemicals with nonhazardous ones,

(ii) Changing the work processes and activities to reduce or eliminate work exposure,

(iii) Installation of exhaust ventilation systems,

(iv) Implementing effective housekeeping at the workplace, and

(v) Providing appropriate personal protective equipment [2]

\section{Framework of the CHRA Tool}

The first step in developing the CHRA tool is to identify the current methodology of risk assessment practiced in Malaysia as well as in the global scale. For each step proposed in the CHRA tool, exposure parameters and relevant calculations are collected and used to develop this tool using VB. A constructed framework of the proposed CHRA tool is shown in Figure 1. The first step in conducting CHRA is the hazard identification stage to determine the hazard rating of the exposed chemical. When the hazard of the chemical has been assessed, the exposure rating is determined on the basis of the frequency or duration of exposure and the magnitude of exposure. The value of hazard rating and exposure rating will then determine the risk rating of the chemical. By obtaining the risk rating, the software will be able to assess the adequacy of the control measures and will conclude the assessment.

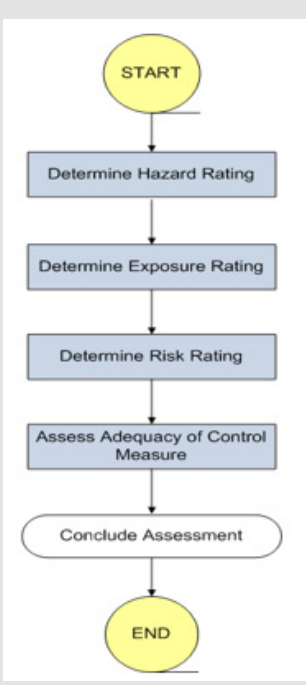

Figure 1: Framework of chemical health risk assessment tool.

Hazard Rating Determination: The first step is to collect hazard information on the types of health effects and the diseases caused by the chemical. Hazard information can be found in numerous sources such as the CSDS that offers useful information such as the hazards identification, toxicological information, chronic and acute toxic effects. The occupational safety and health (classification, packaging, and labeling of hazardous chemicals) 1997 (OSHA-CPL Regulations 1997) categorized chemicals that are hazardous to health based on systemic and local effects into two groupings. These groupings are intended to differentiate the route of exposure of the chemicals whether through inhalation, absorption, or ingestion. Inhalation and ingestion cause systemic effects (Group 1, Appendix 1 in the supplementary materials), whereas absorption, either through the skin or eyes, most of the time causes local effects (Group 2, Appendix 1). The details of each grouping are available in OSHA-CPL Regulations 1997. These details would then be used by the tool to assess the exposure and assign a hazard rating. Appendix 2 presents the hazard rating based on the adverse health effects of the chemical. Classification of the risk based on the adverse health effects of the chemical and classification of the risk based on risk phrases assigned to the hazardous chemical under the OSH-CPL Regulations of 1997 are presented in Appendix 3. The meaning of the codes of the risk phrases are described in Appendix 4. 
Exposure Rating Determination: The estimation of the degree of exposure is primarily based on the following parameters:

i. Frequency of exposure (acute effects) or

ii. Duration of exposure (chronic effects)

iii. Magnitude of exposure

An exposure rating is assigned based on the frequency-duration rating, and the magnitude rating (Table 1 ) is assigned as described in DOSH [23].

Table 1: Exposure rating [23].

\begin{tabular}{|c|c|c|c|c|c|c|}
\hline \multicolumn{2}{|c|}{} & \multicolumn{6}{|c|}{ Magnitude Rating } \\
\cline { 2 - 7 } \multicolumn{2}{c|}{} & $\mathbf{1}$ & $\mathbf{2}$ & $\mathbf{3}$ & $\mathbf{4}$ & $\mathbf{5}$ \\
\hline \multirow{2}{*}{$\begin{array}{c}\text { Fre- } \\
\text { quency } \\
\text { Rating/ } \\
\text { Dura- } \\
\text { tion } \\
\text { Rating }\end{array}$} & 1 & 1 & 2 & 2 & 2 & 3 \\
\cline { 2 - 7 } & 2 & 2 & 2 & 3 & 3 & 4 \\
\cline { 2 - 7 } & 5 & 2 & 3 & 3 & 4 & 4 \\
\hline
\end{tabular}

Frequency of Exposure Determination: The frequency of exposure is used to assess the likelihood of the occurrence of acute effects (short-term exposures). Estimation is made on the basis of the observation of work activities and workers and the management feedback. Frequency rating is calculated based on the frequency of exposure as described in Appendix 5 [23].

Duration of Exposure Determination: The duration rating is used to assess chronic (long-term) exposure based on the total duration of exposure in hours as described in Appendix 6 [23]. Estimating the intensity or the magnitude rating can be done either quantitatively or qualitatively. Quantitative exposure is evaluated for inhalation exposures where air sampling data for exposed employees are available. In case the exposure data are limited or unavailable, the evaluation needs to be performed qualitatively.

Magnitude Rating Determination: There are two possible methods to determine the exposure magnitude or intensity, i.e., quantitatively or qualitatively, as illustrated by the scenarios in Figure 2 .

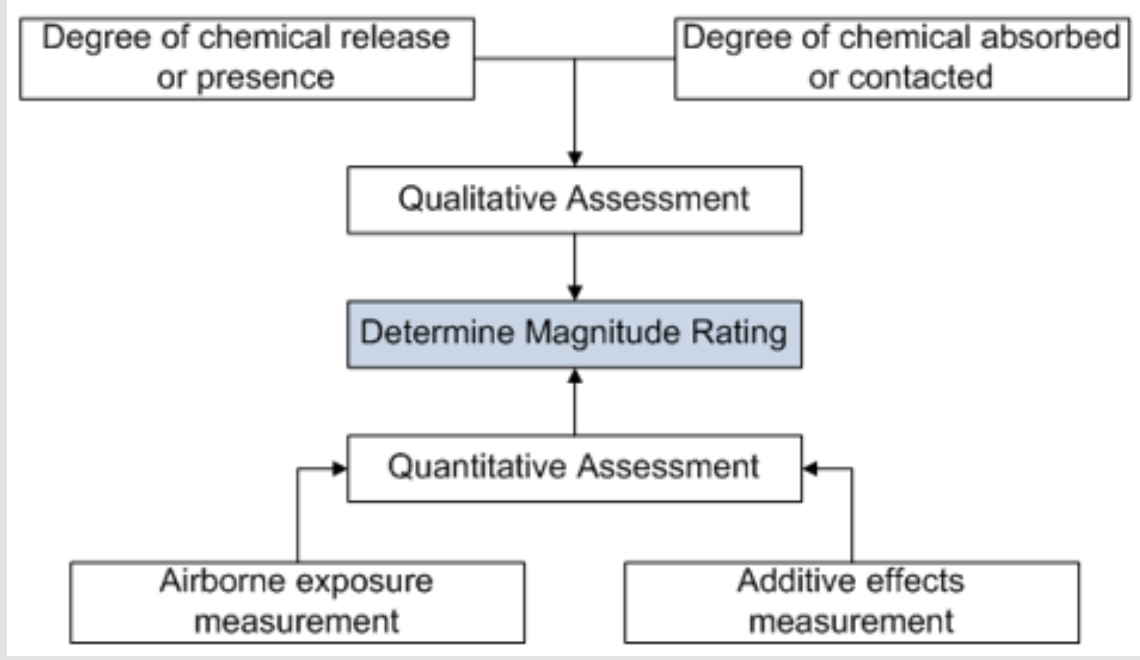

Figure 2: Summary of determining magnitude rating.

Quantitative Evaluation: For chemicals with acute effects, the magnitude rating is assigned based on the measurement result portion to the ceiling limit or the maximum exposure, whichever results in a higher exposure rate. However, for chemicals with chronic exposures, the time period for the evaluation period is 1 week and will depend on exposure for 8-h time-weighted average time (TWA) [23]. The magnitude rating is assigned based on the ratio of the TWA to the 8-h TWA limit as described in Appendix 7.

The American Conference of Governmental Industrial Hygienists (ACGIH) proposed Eq. (1) to calculate TWA [29]:

$$
T W A=\frac{C_{1} T_{1}+C_{2} T_{2}+\ldots+C_{n} T_{n}}{T_{1}+T_{2}+\ldots+T_{n}}
$$

where,

$\mathrm{C}=$ concentration of the sample, and

$\mathrm{T}=$ sampling time

For employees who are exposed to a particular chemical at several job tasks, the 8-h exposure estimation can be determined using Eq. (2) as follows: 


$$
T W A=\frac{D_{1} C_{1}+D_{2} C_{2}+\ldots+D_{n} C_{n}}{D_{1}+D_{2}+\ldots+D_{n}}
$$

where,

$\mathrm{C}=$ average concentration for each task, and

$\mathrm{D}=$ average duration for each task.

Employees who are exposed to two or more unknown materials that act independently of each other must be treated as acting additionally and a "combined PEL" can be estimated but should not exceed unity (Appendix 8).

Qualitative Estimation of the Magnitude of Exposure: The magnitude of exposure is evaluated based on the estimated absorbed dose of the chemical through inhalation and skin absorption. The calculation required should take into consideration the degree of chemical release (Appendix 9) and the degree of chemical absorbed (Appendix 10). When the qualitative estimation of the magnitude of exposure is made based on the degree of chemical released and absorbed, the information is used to estimate its magnitude rating (Appendix 11).

\section{Risk Rating Determination}

Risk is evaluated as either "significant" or "insignificant." Risk ratings located above the dotted line in Figure 3 are considered as not significant, whereas those located below the dotted line are considered as significant risks. Based on Figure 3, control strategies can be identified and prioritized. For the purpose of prioritizing action to control risks, the following two categories can be assigned under significant risk:

\begin{tabular}{|c|c|c|c|c|c|c|}
\hline & & \multicolumn{5}{|c|}{ Exposure Rating } \\
\hline & & 1 & 2 & 3 & 4 & 5 \\
\hline \multirow{5}{*}{ 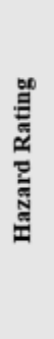 } & 1 & 1 & 2 & 2 & 2 & 3 \\
\hline & 2 & 2 & 2 & 3 & 3 & 4 \\
\hline & 3 & 2 & 3 & & 4 & 4 \\
\hline & 4 & 2 & 3 & & 4 & \\
\hline & 5 & 3 & 4 & 4 & & \\
\hline
\end{tabular}

Figure 3: Risk rating [23].

i. Category 1: Risks is to be controlled to below the PEL or to as low as reasonably practicable where no limits are specified. ii. Category 2: The hazardous chemicals should be eliminated as these are considered as intolerable risks. If this is not possible, then the hazardous chemicals should be substituted with less hazardous ones.

\section{Control Measures}

The risk resulting from the use of hazardous chemicals can be controlled in the following order [23]:

a) Elimination of hazardous chemicals from the workplace,

b) Substitution of hazardous chemicals with less hazardous ones,

c) Total enclosure of the process and handling systems,

d) Working isolation to control the emission of hazardous chemicals,

e) Modification of the process parameters,

f) Minimizing workplace hazards using engineering control equipment and PPE,

g) Practicing safe work systems and adopting practices that eliminate or reduce health risks, and

h) Providing certified PPE.

The existing control measures must be evaluated. The following factors must be taken into consideration:

i. Suitability,

ii. Use,

iii. Effectiveness, and

iv. Maintenance.

Existing control measures are considered as adequate if they meet the following requirements [23]:

a) The measures are appropriate to protect workers, taking into account the nature of the work, the toxicity of the chemical, the routes of entry of the chemical, and not compromising the health of workers;

b) The measures are suitable for protecting the employees, taking into consideration the nature of work, the physical form and toxicity of the chemical, and the routes of entry of the chemical;

c) They are used according to the instructions and recommendations of manufacturers;

d) They are effective in preventing or minimizing the risk from exposure; and

e) They are in good working condition and regularly maintained. 
The conclusions of the assessment based on the risk decision and the assessment of existing control measures are summarized in Table 2.

Table 2: Conclusions of the assessment [23].

\begin{tabular}{|c|c|c|}
\hline Risk Decision & $\begin{array}{c}\text { Adequacy of Control } \\
\text { Measures }\end{array}$ & Conclusion \\
\hline Risk Not Significant & - & $\mathrm{C} 1$ \\
\hline Risk Significant & Adequate & $\mathrm{C} 2$ \\
\cline { 2 - 4 } & Not Adequate & $\mathrm{C} 3$ \\
\hline $\begin{array}{c}\text { Insufficient Informa- } \\
\text { tion }\end{array}$ & - & $\mathrm{C} 4$ \\
\hline $\begin{array}{c}\text { Uncertain about } \\
\text { Exposure }\end{array}$ & - & $\mathrm{C} 5$ \\
\hline
\end{tabular}

\section{Software Development}

The application Microsoft Visual Basic 6.0 (VB 6.0) was used in developing the CHRA tool. VB is relatively simple to learn due to its graphical development features. The programming codes used are built using the VB programming language (source code).

The development process of this software has been divided into the following four different stages: a. Planning the application

b. Building the graphical user interface (GUI)

c. Writing the computer program

d. Software validation and verification

Planning the Application: The first step in application planning is identifying the various tasks that the application needs to perform. The second step is to determine how these tasks are logically related and to identify the objects to which each task will be assigned. This is followed by classifying the events required to trigger an object into executing its assigned tasks. Finally, a sketch of the GUI is prepared. A flowchart of the CHRA tool is presented in Figure 4. The application is designed based on object-oriented programming. It has been designed using multiple GUIs. The GUI is easy to use and users can easily insert data into the tool to be analyzed by the software using just a few clicks. Figure 5 depicts the main interface of the developed tool. Utilizing the GUI implemented in VB, users can input the necessary data into the tool and obtain a risk rating followed by an assessment of the adequacy of the control measures for that particular exposure.

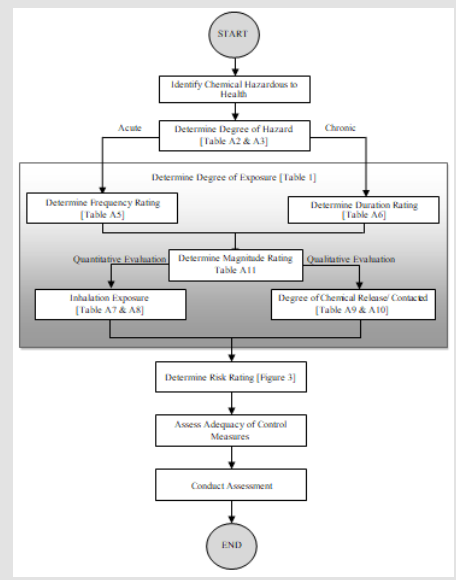

Figure 4: Flowchart of the chemical health risk assessment tool.

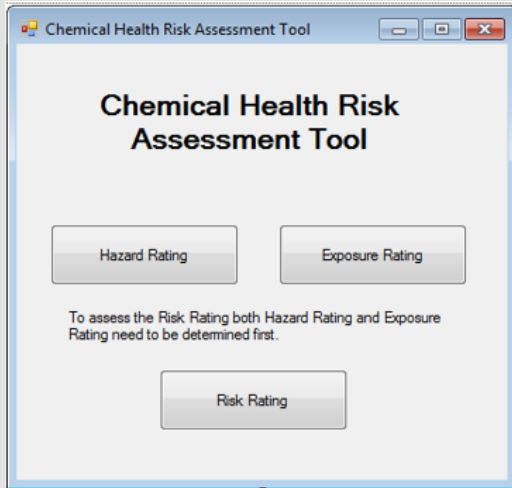

Figure 5: Software introduction interface. 
Writing the Computer Program: The application code is written using the standard Microsoft Visual Basic 6.0 and distributed in object format with the source code. After generating the interface for the application, it is necessary to write the code that defines the application's behavior.

Software Verification and Validation: Verification and validation of computational simulations are the most important steps to build confidence and quantify the results. Verification assesses the accuracy of a solution to a computational model, whereas validation implies the assessment of the accuracy of a computational simulation in comparison with experimental data.

\section{CHRA Tool Features and Applications}

\section{Software Introduction Interface}

After accessing the software, the interface (Figure 5) would appear on the screen. The user would then be able to choose between determining the hazard rating or the exposure rating first. When the user selects his/her choice, the application will lead directly to the chosen rating interface. If the user chooses the risk rating command button before completing the assessment of the hazard rating and the exposure rating first, the software will generate an error prompt message to remind the user to complete the said assessment (Figure 6).

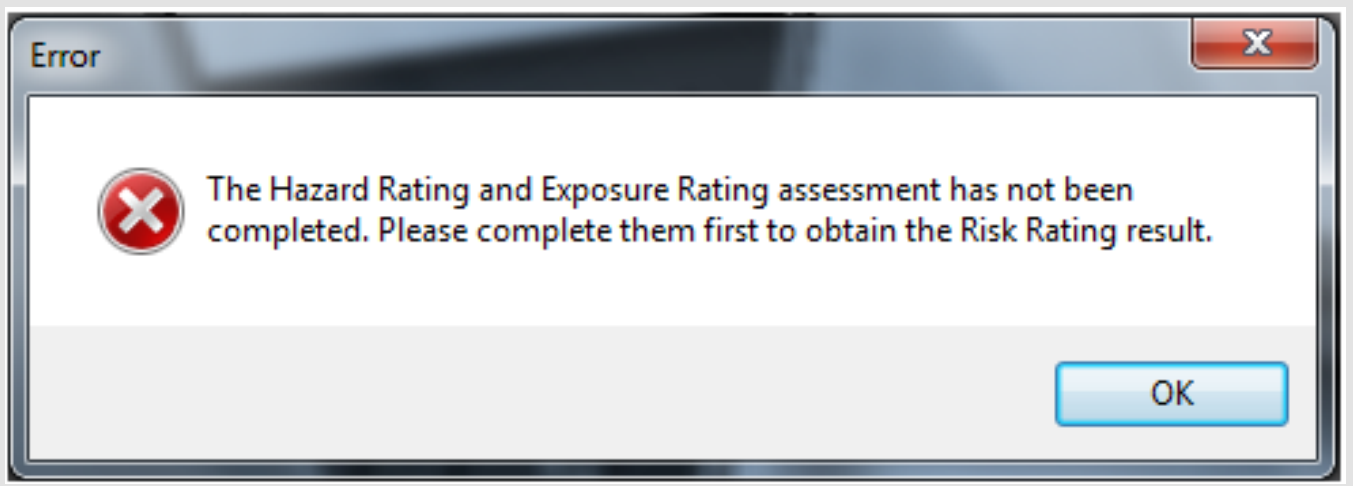

Figure 6: Error message generated by the CHRA tool.

\section{Hazard Rating Interface}

Once the user has chosen the hazard rating on the introduction interface, the user will be asked to select between two methods of assessing the hazard rating, which are based on hazard categories or risk phrases.

Hazard Rating Based on Hazard Categories Interface: If the hazard category is chosen, the application will gain access to the hazard rating based on hazard categories interface and displays it (Figure 7). The user will have to fill in the name of the chemical first before gaining access to the choices. The user can choose only one option. After choosing the option and filling in the necessary fields (if necessary), the user can click the "Result" button to obtain the hazard rating. If the user fails to do so, an error message will be generated to remind the user to complete the assessment (Figure 8).

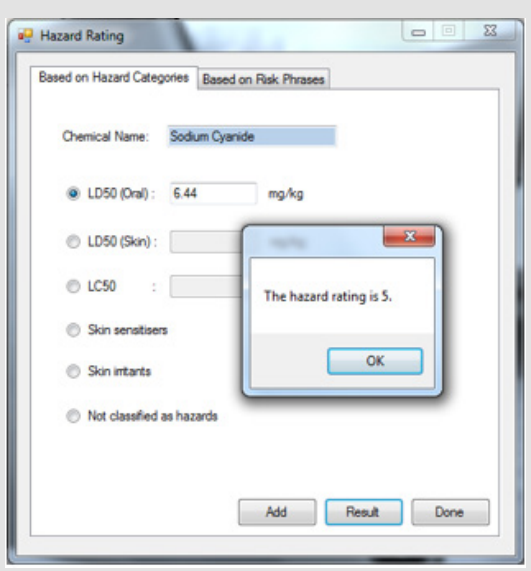

Figure 7: Hazard rating based on hazard categories interface. 


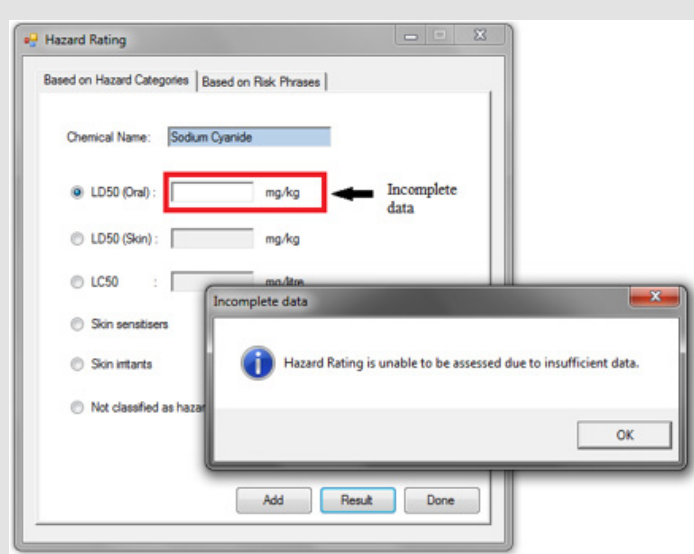

Figure 8: Reminder to complete the fields.

Hazard Rating Based on Risk Phrases Interface: If a risk phrase is chosen, the application will gain access to the hazard rating based on risk phrases interface and will then display it (Figure 9). The user can choose only one option. The user will have to fill in the name of the chemical that the workers are being exposed to as well as its required properties. When all the necessary fields have been filled in, the user will be able to click on the "Result" com- mand button to obtain the hazard rating. If the user fails to do so, an error message will be generated by the software to alert the user about the missing field(s) that has not been keyed in (Figure 9). The "Add" command button allows the user to add properties of another chemical in case the user wishes to assess the risk of exposure to a mixture of chemicals. The "Return" command button will bring the user to the introduction interface of the software.

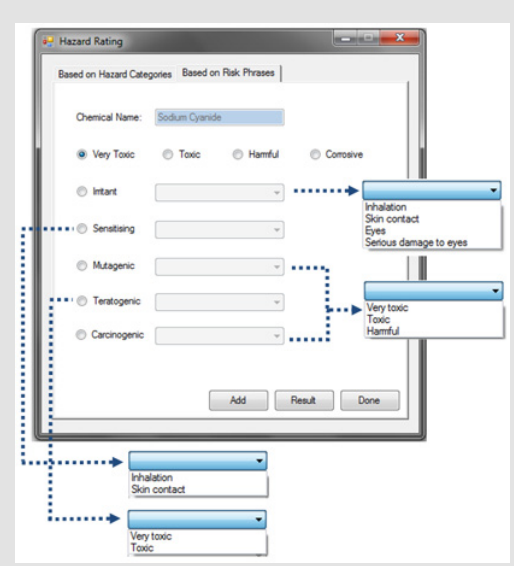

Figure 9: Hazard rating based on risk phrases interface.

\section{Exposure Rating Interface}

Frequency Rating Interface: When the user clicks the exposure rating command button on the general interface (Figure 5), the frequency and duration rating interface will be shown. The user will be able to choose between assessing the frequency of exposure or the duration of exposure. If the frequency of exposure is chosen, the application will gain access to the frequency rating interface (Figure 10). The user will be required to choose any one of the frequency of exposure that best suits the situation.

Duration Rating Interface: If the user chooses to assess the duration of exposure instead, the duration rating interface will be displayed (Figure 11). The user will be required to fill in the val- ue for the number of exposures per week as well as the average duration of exposure. The software will then use these values to calculate the total duration of exposure per week. When the user has filled in all the required fields, he/she can click on the "Next" button to continue with the assessment, i.e., determining the intensity or magnitude rating. If the user fails to do so, clicking the "Next" button will generate an error message to remind the user to fill in the required data (Figure 12). If the "Return" command button is chosen, the user will be directed to the introduction interface.

Intensity or Magnitude Rating Interface: After the assessment of the frequency or duration rating, the user will be asked to assess the intensity or magnitude of exposure. There are two pos- 
sible methods to estimate the exposure intensity or magnitude, either quantitatively or qualitatively.

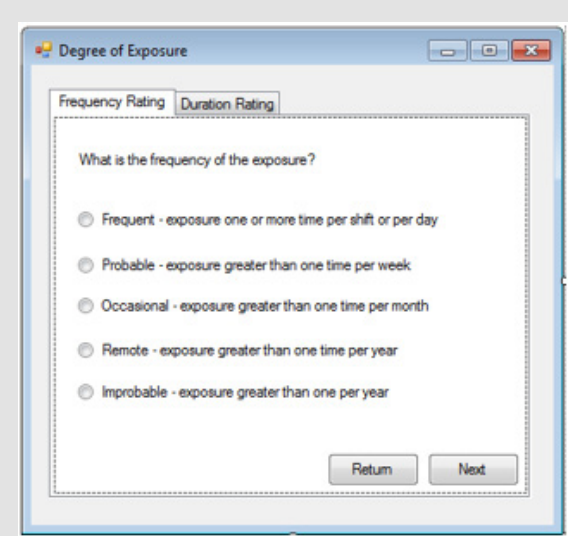

Figure 10: Frequency rating interface.

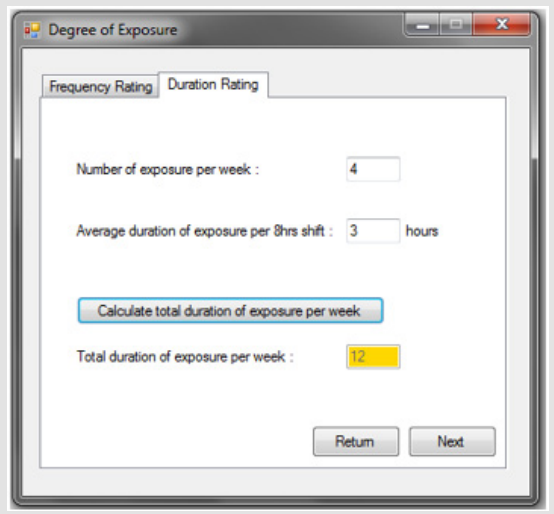

Figure 11: Duration rating interface.

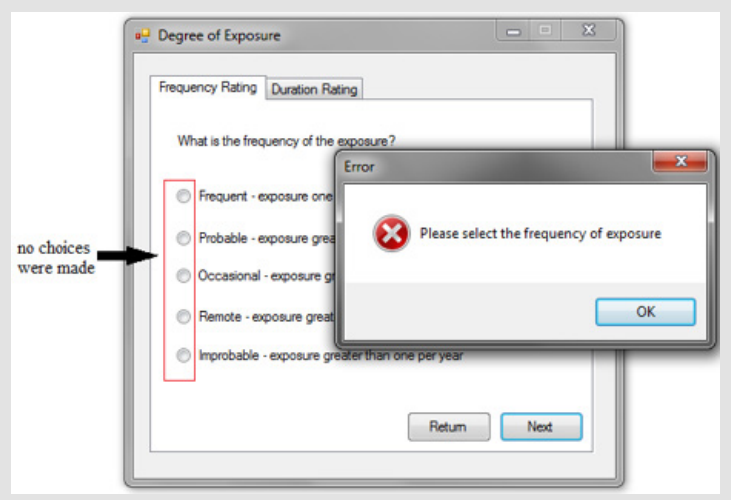

Figure 12: Error message generated.

Quantitative Evaluation Interface: In the quantitative evaluation interface, the user has to key in the concentration of the exposed chemical, the sampling time, and the occupational exposure limit of the said chemical (Figure 13). As the chemical name would have already been provided by the user during the hazard rating assessment, it does not need to be filled in again. If the user has filled in all the required fields, then the user would be able to click the "Result" command button, which will evaluate the exposure rating.
If the user fails to do so, an error message will be generated by the software to remind the user to fill in the required field(s) (Figure 13). If the user does not have the PEL value for the said chemical, the user may click on the hyperlink "List of Chemical's Permissible Exposure Limit," which will open a PDF file containing the list of PEL values for common chemicals.

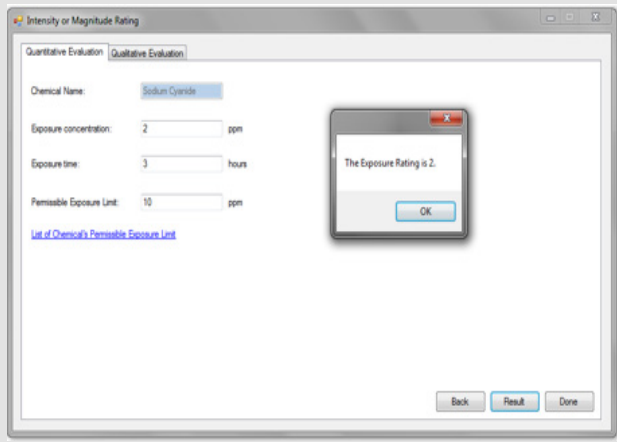

Figure 13: Quantitative evaluation interface.

Qualitative Evaluation Interface: In the qualitative evaluation interface, the user is required to determine both the degree of chemical release or presence and the degree of chemical absorbed or contacted (Figure 14). The degree of chemical release or presence is divided to three categories, including low, moderate, and high. The user is given the choice to choose only one of the categories that best suits the current situation. The user would be able to reconfirm the assessment by checking the solvent drying time (Appendix 12) and the solvent odor thresholds (Appendix 13) through the link provided at the bottom of the interface. The degrees of chemical absorbed or contacted are also divided to three categories, viz., low, moderate, and high (Appendix 14). The user can choose only one that best describes the situation at hand. A link is provided for more information on determining the degree of physical activities and breathing rate (Appendix 15) to assist the user on deciding which category to choose. Both these assessments will be used to determine the intensity or magnitude rating. Once the user has chosen the category for both degrees, he/she will be able to click on the "Result" button command to obtain the exposure rating. If no choices are made, the software will generate an error message prompting the user to complete the assessment (Figure 15). If the "Back" command button is clicked, it will return the user to the frequency or duration rating interface.

\section{Risk Rating}

After the completion of both the hazard rating and the exposure rating assessment, the user would be able to click on the Risk Rating button on the general interface. This will prompt the software to produce a risk rating report based on the assessment that the user has performed. The report will be presented as a Microsoft Office Word document, which can be saved depending on the user's preferences (Figure 15). 


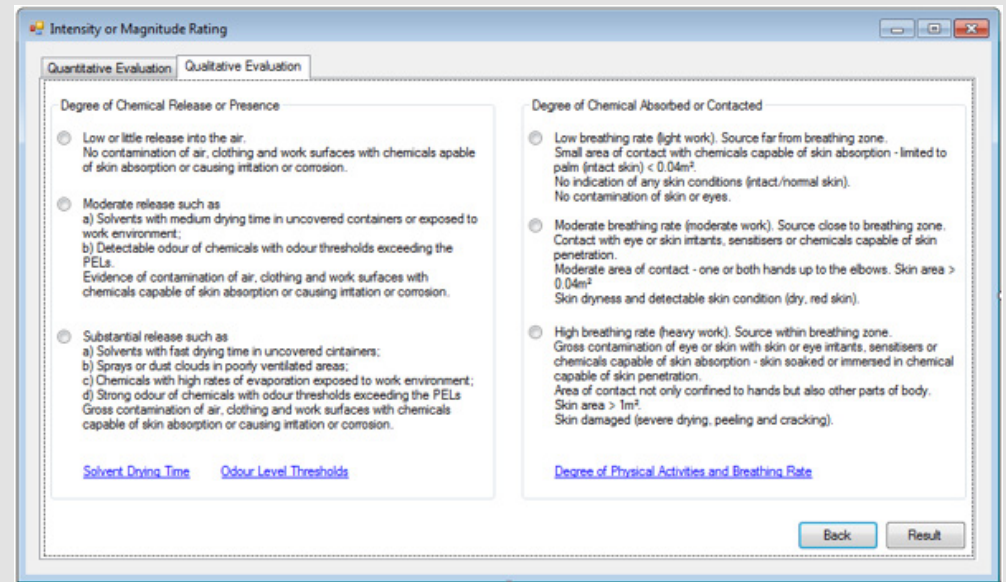

Figure 14: Qualitative evaluation interface.

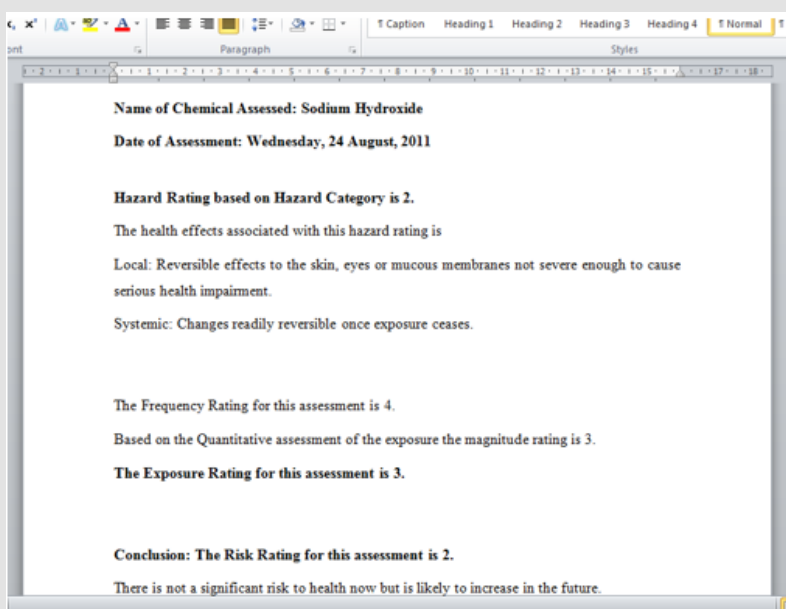

Figure 15: Risk rating assessment report.

\section{Case Study}

The CHRA tool software can be applied for assessing the risk rating of an exposure to a hazardous chemical. To our knowledge, there is no software that has been developed to be compared with the CHRA tool. Therefore, a case study was done to compare the result of the CHRA tool with the current risk rating assigned through manual CHRA. The chosen case study was to assess the health risk of exposure to chemicals found in chemical laboratory at Universiti Teknologi PETRONAS. Currently, the laboratory is equipped with appropriate ventilation system and appropriate signs to identify specific hazards within an area and the use of PPE. The CHRA tool software generated a risk rating of 2 where the risk is found to be significant and could increase in the future due to the following aspects:

a. Undetected decline in the efficiency of control measures.

b. Plant, equipment, PPE, or system failure.

c. Human error due to lack of awareness or insufficient training.

d. A significant increase in the quantity of chemicals used.

It can be concluded that the results obtained from the CHRA tool are in good agreement with the current risk rating assigned to this laboratory.

\section{Conclusion}

Workers are exposed to chemicals on a daily basis, especially in processing industries. This exposure can cause minor and major effects to the health of the workers. A CHRA is required at all workplaces to assess the risk to health from exposure to chemicals at work. However, it is difficult to apply this manual assessment primarily because of the following reasons:

(i) Calculations done manually can cause absolute error,

(ii) Manually browsing through the CSDS to identify hazard information for the candidate chemicals can be tedious, thus resulting in difficulty to keep track of them, 
(iii) A CHRA requires step-by-step procedures that must be conducted by an assessor, which would be time-consuming, and

(iv) It requires an assessor who is an expert in the field to perform the assessment manually. For these reasons, the assessment is best conducted using a computerized tool.

This paper describes the stages of the CHRA tool development using the VB programming language. This tool allows the users to assess the risk rating of an exposed chemical through the assessment of its hazard and exposure rating. The hazard rating can be assessed by two methods, hazard rating based on hazard categories and hazard rating based on risk phrases. The exposure rating is obtained by assessing the frequency/duration rating and the intensity or magnitude rating of the exposed chemical. The obtained risk rating provides the user the adequate control measures and steps that must be taken to ensure the safety of all people. The result of the CHRA tool was compared with an existing manual CHRA due to the lack of a currently developed software with similar capabilities. It was confirmed that the results are consistent with no significant deviation. The CHRA tool is practical and feasible because it is user-friendly, can function as a stand-alone application, and it is compatible with all Windows operating systems. Furthermore, the Malaysian standards and regulations are incorporated into the developed tool, whereby users can compare their results with the risk tolerability limit for Malaysia.

\section{Acknowledgments}

The authors acknowledge the support provided by Univeristi Teknologi PETRONAS for completing this work. Mohanad El-Harbawi extends the appreciation to the Deanship of Scientific Research at King Saud University for funding this work through research group no. RGP-303.

\section{References}

1. Manassaram DM, Orr MF, Kaye WE (2003) Hazardous substances events associated with the manufacturing of chemicals and allied products. Journal of Hazardous Materials 104(1-3): 123-135.

2. Plog BA, Quinlan PJ (2002) Fundamentals of Industrial Hygiene, $\left(5^{\text {th }}\right.$ Edn.,), National Safety Council, USA.

3. In: MacGregor S, Thom B (Eds.,) (2020) Risk and Substance Use: Framing Dangerous People and Dangerous Places. Routledge.

4. Bisesi MS (2003) Industrial Hygiene Evaluation Methods. (2 ${ }^{\text {nd }}$ Edn.,). Lewis Publishers, USA.

5. Bláhová L, Nováková Z, Večeřa Z, Vrlíková L, Dočekal B, Dumková J, et al. (2020) The effects of nano-sized PbO on biomarkers of membrane disruption and DNA damage in a sub-chronic inhalation study on mice. Nanotoxicology 14(2): 214-231.

6. Dikshith TSS (2008) Safe use of chemicals: a practical guide. CRC Press.

7. (2012) WHO (World Health Organization). Poison prevention and Management. International Programme on Chemical Safety. World Health Organization.

8. (2004) WHO (World Health Organization). Declaration: fourth Ministerial Conference on Environment and Health, Budapest, Hungary, 23-25 June 2004 (No. EUR/04/5046267/6). Copenhagen: WHO Regional Office for Europe.
9. Anderson EL, Albert RE (1999) Risk Assessment and Indoor Air Quality, Lewis Publishers, USA.

10. Arunraj NS, Maiti J (2009) A methodology for overall consequence modeling in chemical industry. Journal of Hazardous Materials 169(13): 556-574

11. Gerba CP (2019) Risk assessment. In Environmental and pollution science, ( $3^{\text {rd }}$ Edn.). . Academic Press. pp. 541-563.

12.(1993) Extoxnet (The Extension Toxicology Network. Toxicology Information Briefs. Risk Assessment Background.

13.(1999) WHO (World Health Organization). Environmental Health Criteria 210: Principles for the Assessment of Risks to Human Health from Exposure to Chemicals. Geneva: World Health Organization.

14. Klapacz J, Gollapudi BB (2020) Considerations for the use of mutation as a regulatory endpoint in risk assessment. Environmental and molecular mutagenesis 61(1): 84-93.

15. (1983) US-NAS National Academy of Science. Risk Assessment in the Federal Government: Managing the Process. National Research Council, Committee on the Institutional Means for Assessment of Risks to Public Health. Washington DC, National Academy Press, pp. 1-50.

16. Somers E (1987) Making decisions with numbers. Regul Toxicol Pharmacol 8: 35-42.

17. (1989) HSE. Health and Safety Executive. Quantified Risk Assessment: Its Input to Decision Making. HM Stationery Office.

18. Scala RA (1991) Risk assessment. In: Amdur MO, Doull J, Klaasen CD (Eds.)). Casarett and Doull's Toxicology. ( $4^{\text {th }}$ Edn.), New York, Oxford, Beijing, Frankfurt, Sao Paulo, Sydney, Tokyo, Toronto, Pergamon Press, pp. 985-996.

19. Ballantyne B, Marrs T, Turner P (1993) General and Applied Toxicology. Vol. 2, Stockton, Stockton Press, pp. 1069-1158.

20. Somers E (1993) Perspectives on Risk Management. Paper presented at Managing Risks to Life and Health Meeting, 18 October, Ottawa, Royal Society of Canada.

21. (1996) EC. Technical Guidance Document in Support of the Commission Directive 93/67/EEC on Risk assessment for New Notified Substances and Commission Regulation 1488/94/EEC on Risk assessment for Existing Chemicals. Brussels, European Commission.

22. (2009) EU-OSH. European Agency for Safety and Health at Work Annual Report 2009.

23. (2000) DOSH (Department of Occupational Safety and Health) Assessment of the Health Risks Arising from the Use of Hazardous Chemicals in the Workplace. Ministry of Human Resources, Malaysia.

24. In: Deelstra H, Fondu M, Ooghe W, Van Havere R (Eds.,). (1991) Food policy trends in Europe: nutrition, technology, analysis and safety. Elsevier.

25. Boss MJ, Day DW (2001) Air Sampling and Industrial Hygiene Engineering. CRC Press LLC, USA.

26. Leeuwen CJ, Vermeire T, Vermeire TG (2007) Risk Assessment of Chemicals: An Introduction. Springer, USA.

27. Aven T (2016) Risk assessment and risk management: Review of recent advances on their foundation. European Journal of Operational Research 253(1): 1-13.

28. Griffin RD (2009) Principles of Hazardous Materials Management, $\left(2^{\text {nd }}\right.$ Edn.,). Taylor and Francis Group, LLC, USA.

29. (1997) American Conference of Governmental Industrial Hygienists (ACGIH). Guide to Occupational Exposure Values. ACGIH, Inc, Cincinnati, $\mathrm{OH}$ 
ISSN: 2574-1241

DOI: 10.26717 BJSTR.2020.28.004669

Mohanad El-Harbawi. Biomed J Sci \& Tech Res

\section{(c) Commons Attribution 4.0 License}

Submission Link: https://biomedres.us/submit-manuscript.php

\begin{tabular}{ll} 
BIOMEDICAL & Assets of Publishing with us \\
RESEARCHES & - Global archiving of articles \\
- Immediate, unrestricted online access & - Rigorous Peer Review Process \\
\hline ISSN: 2574-1241 & - Authors Retain Copyrights \\
&
\end{tabular}

